\title{
The coupling between the solar wind and proton fluxes at GEO
}

\author{
R. J. Boynton ${ }^{1}$, S. A. Billings ${ }^{1}$, O. A. Amariutei ${ }^{2}$, and I. Moiseenko ${ }^{3}$ \\ ${ }^{1}$ ACSE, University of Sheffield, Sheffield, UK \\ ${ }^{2}$ Finnish Meteorological Institute, Helsinki, Finland \\ ${ }^{3}$ Space Research Institute, RAS, Moscow, Russian Federation \\ Correspondence to: R. J. Boynton (r.boynotn@sheffield.ac.uk)
}

Received: 11 March 2013 - Revised: 15 July 2013 - Accepted: 9 August 2013 - Published: 2 October 2013

\begin{abstract}
The relationship between the solar wind and the proton flux at geosynchronous Earth orbit (GEO) is investigated using the error reduction ratio (ERR) analysis. The ERR analysis is able to search for the most appropriate inputs that control the evolution of the system. This approach is a black box method and is able to derive a mathematical model of a system from input-output data. This method is used to analyse eight energy ranges of the proton flux at GEO from $80 \mathrm{keV}$ to $14.5 \mathrm{MeV}$. The inputs to the algorithm were solar wind velocity, density and pressure; the Dst index; the solar energetic proton (SEP) flux; and a function of the interplanetary magnetic field (IMF) tangential magnitude and clock angle. The results show that for lowest five energy channels ( 80 to $800 \mathrm{keV}$ ) the GEO proton fluxes are controlled by the solar wind velocity with a lag of two to three days. However, above $350 \mathrm{keV}$, the SEP fluxes, accounts for a significant portion of the GEO proton flux variance. For the highest three energy channels ( 0.74 to $14.5 \mathrm{MeV}$ ), the SEPs account for the majority of the ERR. The results also show an anisotropy of protons with gyrocenters inside GEO and outside GEO, where the protons inside GEO are controlled partly by the Dst index and also an IMF-clock angle function.
\end{abstract}

Keywords. Magnetospheric physics (solar windmagnetosphere interactions)

\section{Introduction}

The Van Allen radiation belts, first discovered by Explorer I (van Allen, 1958), are regions of the magnetosphere that are inhabited by highly energetic particles trapped by the Earth's magnetic field. These trapped particles can significantly increase the probability of detrimental effects to the onboard satellite systems and can even lead to permanent hardware damage (Roeder and Fennell, 2009). The trapped energetic electrons have energies ranging from tens of $\mathrm{keV}$ up to a few $\mathrm{MeV}$ and span from, approximately, $1.2 R_{\mathrm{E}}$ to $7.8 R_{\mathrm{E}}$ in an inner and an outer radiation belt, separated by a slot region. On the other hand, the trapped energetic protons form one belt, with the higher energy protons $(>10 \mathrm{MeV})$ occupying lower altitudes and the lower energy protons $(>1 \mathrm{MeV})$ at higher altitudes extending beyond geosynchronous Earth orbit (GEO).

High energy solar energetic protons (SEPs) are known to have access to GEO. Paulikas and Blake (1969), using data from GEO satellite ATS 1 and measurements from outside the magnetosphere, showed that during magnetically quiet times, protons with energies $>20 \mathrm{MeV}$ have free access to the geosynchronous regions of the magnetosphere as the theory suggested. However, the energies lower $20 \mathrm{MeV}$ were also shown to have access to the magnetosphere, in contrast to the theories at the time. Paulikas and Blake (1969) pointed out that these $<20 \mathrm{MeV}$ protons mainly occurred in the nightside sector and concluded that the access of the $5-21 \mathrm{MeV}$ protons is governed by the structure and fluctuations in the magnetic field well outside the GEO. Blake et al. (1974) showed that these protons have access to the GEO through the flanks of the magnetosphere and are then temporally trapped with a lifetime of a few drift periods. Observations on polar orbiting satellites have shown that the access of $>1 \mathrm{MeV}$ solar protons to lower L-shells is correlated with Dst, AE and dynamic pressure (Ivanova et al., 1985). storm sudden commencements (SSCs) associated with coronal mass ejections (CMEs) are known to inject the $>10 \mathrm{MeV}$ protons into the magnetosphere (Vampola and Korth, 1992; Blake et al., 1992; Li et al., 1993; Hudson et al., 1995). Richard et al. (2002) studied the importance 
of the interplanetary magnetic field (IMF) orientation on the access of SEPs and found that a southward IMF condition resulted in the largest access to the magnetosphere. More recently, a case study of an SEP event and the resulting eastwest anisotropies of the proton flux at GEO was performed by Rodriguez et al. (2010). They found that access of the SEPs was increased with high dynamic pressure $(>10 \mathrm{nPa})$ for protons inside GEO. While for low dynamic pressure, repeated undulations of the proton and ion fluxes inside GEO were found to correlated the auroral electrojet indices.

For this study, the objective was to identify solar wind parameters (including the proton fluxes within the solar wind) that control the various energies of the proton fluxes at GEO. This was achieved by the use of an advanced system identification technique: Nonlinear AutoRegressive Moving Average with eXogenous inputs (NARMAX) (Leontaritis and Billings, 1985a, b). This method is able to determine the most appropriate inputs for a system by the use of the error reduction ratio (ERR). In Sect. 2, the methodology of the NARMAX ERR analysis is discussed in more detail along with the data used in this study. The inputs chosen are then considered and the results are shown in Sect. 3. Discussions and conclusions are in Sects. 4 and 5, respectively.

\section{The ERR analysis}

The NARMAX approach is one of the most advanced techniques in the field of system science. It is a black box modelling methodology, similar to neural networks, and can automatically deduce a dynamical equation that governs the evolution of the system from input and output data. However, unlike neural networks, the resulting model can be a polynomial, which is physically interpretable. This is the major advantage of NARMAX over neural networks. While neural networks lead to the inputs linked to the output through a maze of neurons, which is very difficult to understand, the polynomial that results from NARMAX can be, in some sense, reverse engineered to gain physical understanding about the system and the processes involved. Examples of this in the field of space physics can be found in various studies. Boaghe et al. (2001) identified a NARMAX model for the Dst index, then the dominant nonlinear characteristics were studied by mapping this model into the frequency domain to produce a generalized frequency response function. This revealed evidence for the existence of energy storage processes that involve multi-wave coupling. Balikhin et al. (2001) performed a similar study that targeted the processes of energy loading for the Dst index, finding no support for models that assume a time delay storage of energy. Boynton et al. (2011) used the same methodology as the one employed in this study to obtain a solar wind magnetosphere coupling function for the Dst index. Balikhin et al. (2010) then explained this coupling function analytically, using the geometry of dayside reconnection between the solar wind and the magnetosphere. Another example is Boynton et al. (2013) employing this approach on the election flux for 14 energies, ranging from 24 to $3.5 \mathrm{MeV}$. One of the results in this study showed a relationship between the energy of the electron flux and the time delay in which it takes the solar wind velocity to effect the flux. Although not a new result, the ERR analysis allowed for the quantification of this time delay and from this Balikhin et al. (2012) could show, by solving the energy diffusion equation (Horne et al., 2005; Schulz and Lanzerotti, 1974), that acceleration of the electrons was too fast to be explained solely by local diffusion due to the wave-particle interaction.

There are three stages to obtain a model by the NARMAX algorithm. The first, model structure detection, is to find the terms that are most appropriate for the system (Billings et al., 1989). The second stage estimates the coefficient for these terms and, lastly, the model is validated (Billings and Voon, 1986). In this study the structure detection stage is employed to find the most appropriate combination of solar wind terms that govern the evolution of the proton flux. In the structure detection stage of the NARMAX algorithm, the chosen inputs, in this case the solar wind parameters, are cross-coupled to a specified degree of nonlinearity. This leads to numerous different combinations of the inputs, many of which will have no effect on the system output (the proton fluxes). Therefore, the ERR is then employed to determine the amount of dependent variable variance explained by each of the combinations of the inputs and is thus able to find and assess the most appropriate combination of solar wind parameters.

\subsection{Instrumentation}

For this study, the proton flux data are from the Geostationary Operational Environmental Satellite (GOES). GOES 13 was the first to introduce the new MAGnetospheric Proton Detector (MAGPD) instrument, which has 5 energy channels ranging from 80 to $800 \mathrm{keV}$ (Hanser, 2011). These protons, for the $90^{\circ}$ pitch angle, will have a gyroradii ranging from 0.16 to $0.51 R_{\mathrm{E}}$ with a low magnetic field value of $40 \mathrm{nT}$. The MAGPD instrument has 9 telescopes, one directed radially outwards from Earth centered on a $90^{\circ}$ pitch angle, 4 looking north-south at $35^{\circ}$ and $70^{\circ}$ relative to anti-Earth, and 4 more looking east-west at the same angles, each with a $30^{\circ}$ full cone angle. In this study, only Telescope 1 was employed, since the observed protons will be closest to GEO and the small gyroradii means there should not be much difference between the origin of the east and west protons with $90^{\circ}$ pitch angle.

For higher energy protons, three energy channels, from the Energetic Proton Electron and Alpha Detector (EPEAD) on GOES 13, were used in this study to see if the access of SEP had any dependance on solar wind conditions. The energies of these channels ranged from 0.74 to $14.5 \mathrm{MeV}$. The EPEAD have 2 telescopes, one directed eastward, the other westward. Therefore, the eastward orientated telescope will 
Table 1. Results of the ERR analysis. Shows top three terms with the ERR.

\begin{tabular}{llrlrlr}
\hline Proton flux energy & Term 1 & ERR (\%) & Term 2 & ERR (\%) & Term 3 & ERR (\%) \\
\hline $80-110 \mathrm{keV}$ & $V(t-2)$ & 81 & $V \operatorname{Dst}(t-1)$ & 0.493 & $n V(t-3)$ & 0.412 \\
$110-170 \mathrm{keV}$ & $V(t-3)$ & 70 & $p \operatorname{Dst}(t)$ & 1.23 & $n \operatorname{Dst}(t)$ & 0.9 \\
$170-250 \mathrm{keV}$ & $V(t-3)$ & 65.4 & $n \operatorname{Dst}(t)$ & 1.51 & $\operatorname{Dst}^{2}(t)$ & 1.01 \\
$250-350 \mathrm{keV}$ & $V^{2}(t-3)$ & 66.9 & $V^{2}(t-1)$ & 1.6 & $n \operatorname{Dst}(t)$ & 0.935 \\
$350-800 \mathrm{keV}$ & $V^{2}(t-2)$ & 65.4 & $P_{\mathrm{S}}(t)$ & 9.96 & $n V(t-3)$ & 0.765 \\
$0.74-4.2 \mathrm{E} \mathrm{MeV}$ & $P_{\mathrm{S}} V(t)$ & 88.5 & $P_{\mathrm{S}} p(t-1)$ & 7.91 & $P_{\mathrm{S}} n(t)$ & 2.2 \\
$0.74-4.2 \mathrm{~W} \mathrm{MeV}$ & $P_{\mathrm{S}} V(t)$ & 80.7 & $P_{\mathrm{S}} p(t-1)$ & 12.7 & $P_{\mathrm{S}} n(t)$ & 3.15 \\
$4.2-8.7_{\mathrm{E}} \mathrm{MeV}$ & $P_{\mathrm{S}}(t)$ & 98 & $P_{\mathrm{S}} \operatorname{Dst}(t)$ & 0.816 & $P_{\mathrm{S}} n(t)$ & 0.318 \\
$4.2-8.7 \mathrm{~W} \mathrm{MeV}$ & $P_{\mathrm{S}}(t)$ & 97.7 & $P_{\mathrm{S}}^{2}(t)$ & 0.941 & $P_{\mathrm{S}} \operatorname{Dst}(t)$ & 0.761 \\
$8.7-14.5_{\mathrm{E}} \mathrm{MeV}$ & $P_{\mathrm{S}} \operatorname{Dst}(t)$ & 90.7 & $P_{\mathrm{S}} B_{T} \sin ^{6}(\theta / 2)(t)$ & 2.72 & $p \operatorname{Dst}(t)$ & 0.581 \\
$8.7-14.5 \mathrm{~W} \mathrm{MeV}$ & $P_{\mathrm{S}}(t)$ & 94.2 & $P_{\mathrm{S}} \operatorname{Dst}(t)$ & 2.57 & $P_{\mathrm{S}} B_{\mathrm{T}} \sin ^{6}(\theta / 2)(t)$ & 0.989 \\
\hline
\end{tabular}

observe protons inside GEO and the westward will observe protons with gyrocenters outside GEO. For a magnetic field of $40 \mathrm{nT}$, the range gyroradii at $90^{\circ}$ pitch angle is 0.49 to $2.2 R_{\mathrm{E}}$ for these energies and as such the gyrocenters for the east and west protons will have significantly different origins. Therefore, both the east and west telescopes are employed to compare the protons inside and outside of GEO.

As stated in Sect. 1, the inputs for the proton fluxes at GEO included IMF components in GSM coordinates; solar wind flow speed, density and dynamic pressure; SEP fluxes; and geomagnetic indices. Data from the OMNI website were used for the IMF components, speed, density, pressure and geomagnetic indices, while the SEP flux were the verified level 2 data taken from the Electron Proton and Alpha Monitor (EPAM) onboard the Advanced Composition Explorer (ACE) situated at L1.

The data period for this study is 647 days from 1 January 2011 to 8 October 2012. All the data was daily averaged since the fluxes in a GEO will have a daily variation with the magnetic local time (MLT) and the ERR analysis requires the length of input data to be equal to that of the output. However, daily averaging the data will lead to the loss of the shorter timescale dynamics. This is not as much of a problem for most the inputs considered here but is important for the orientation of the IMF. The typical variation of this is of the order of hours and obtaining information about the orientation of the IMF from the daily averaged IMF components may hide the dynamics that occur on a shorter timescale. Therefore, a number of parameters that could account for the variations of the IMF direction that take place on a short timescale (within each day) were calculated from the minute IMF data.

\subsection{Methodology}

The ERR analysis was applied to obtain the solar wind parameters that control each of the 11 energy channels of the proton flux. Each of the energy ranges was employed to be the output, while the IMF components; solar wind velocity, density and pressure; SEP flux; and the geomagnetic indices were used as the inputs. The ERR algorithm was set to search for all combinations of inputs to a second degree of nonlinearity and four lags of the inputs (the input on the current day, $t$, to the lag of the input four days ago $t-4$ ).

\section{ERR analysis of the proton fluxes}

Six inputs were chosen for the analysis, these were the solar wind velocity $V$, density $n$ and pressure $p$; the Dst index; the SEP flux $P_{\mathrm{S}}$; and a function of the IMF and clock angle. Many different functions of the IMF and clock angle were investigated, from simple daily averages to parameters that could account for the amount of southward IMF within each day. These were calculated from the minute IMF data and included: the daily averages of the components $B_{x}, B_{y}$ and $B_{z}$; magnitude $B$; tangential magnitude $B_{\mathrm{T}}=\sqrt{B_{y}^{2}+B_{z}^{2}}$; the southward IMF $\left(B_{\mathrm{S}}=0\right.$ for $B_{z} \geq 0$ and $B_{\mathrm{S}}=-B_{z}$ for $B_{z}<0$ ) (Burton et al., 1975); functions of the tangential IMF and clock angle, $\theta=\tan ^{-1}\left(B_{y} / B_{z}\right), B_{\mathrm{T}} \sin ^{4}(\theta / 2)$ (Kan and Lee, 1979) and $B_{\mathrm{T}} \sin ^{6}(\theta / 2)$ (Boynton et al., 2011; Balikhin et al., 2010); and the fraction of time in the day that the IMF was southward. Including all these functions would immensely increase the number of monomials in the search, which would take a long time to complete. Therefore, the algorithm was run each time using a different IMF parameter, with the $B_{\mathrm{T}} \sin ^{6}(\theta / 2)$ parameter resulting in the highest ERR. It should be noted that the past outputs where not included in the search, since the aim of this study is the quest for the GEO proton flux inputs. These results are shown in Table 1.

The SEP fluxes were the EPAM fluxes which overlapped the energy ranges of the MAGPD and EPEAD fluxes. It should be noted that there is no SEP flux measured by ACE equivalent to the highest channel measured by the MAGPD studied here. The highest energy channel on the ACE EPAM is $1.89-4.75 \mathrm{MeV}$, while the highest energy at GOES, which this work examined, was $8.7-14.5 \mathrm{MeV}$. 
However, by inspecting the ACE proton data, it can be seen that there is a high correlation between the energy ranges of the proton fluxes. Therefore, it was assumed that the 8.7-14.5 MeV SEP flux would be similar to the SEP fluxes at $1.89-4.75 \mathrm{MeV}$. Also, it should be noted that the $4.2-$ 8.7 MeV channel only had a marginal overlap with the SEP fluxes.

Table 1 shows the top three terms selected by the algorithm along with the ERR for each of the energies analysed. The subscript $\mathrm{E}$ and $\mathrm{W}$ indicate the east (inside) and west (outside) directions of the EPEAD.

For the lower five energy channels from the MAGPD, the solar wind velocity account for the majority of the ERR. The velocity from two days prior is the most appropriate term for the $80-110 \mathrm{keV}$ protons, while to a much lesser extent, the Dst index and density also have an influence. With the two higher energy channels of $110-170 \mathrm{keV}$ and $170-250 \mathrm{keV}$, it is the third lag of the velocity that has the highest ERR, although with a reducing ERR as the energy increased. The Dst index, pressure and density also have a minor role. The $250-350 \mathrm{MeV}$ proton flux is controlled by the third lag of the squared velocity. The highest energy channel of the MAGPD is effected most by the $V^{2}$ from two days previous. However, with $10 \%$ of the ERR, the current days SEP flux has a significant influence on the fluxes at GEO.

For the $0.74-4.2 \mathrm{MeV}$ proton fluxes from the EPEAD, both the east (inside) and west (outside) protons are controlled by the same parameters, mainly the SEP flux coupled with the velocity and a small role from the pressure and density. There are minor differences with the $4.2-8.7 \mathrm{MeV}$ protons with, again, the SEP having the most influence with small contribution from Dst and density. For the highest energy channel, the SEP flux coupled with the Dst index has a large influence for the inside protons and the IMF-clock angle function also has a small role.

\section{Discussion}

All the energy levels on the MAGPD instrument are mainly influenced by a lag in velocity or $V^{2}$. Figure 1 shows a scatter plot for the $80-110 \mathrm{keV}$ proton flux and velocity recorded two days prior. This indicates a complex triangular relationship, similar to the electron fluxes (Reeves et al., 2011), where the proton fluxes have a velocity dependant lower limit but are independent in the upper limit. Figure 1 displays a higher and much narrower range of proton fluxes when the solar wind velocity is high compared to when the velocity is low. Therefore, the higher the solar wind velocity the higher the probability of a high proton flux.

The time lags of the velocity or $V^{2}$ is 2 days for the highest and lowest channel of the MAGPD and three days for the other channels. The fact that from the lowest energy there is an increase in time lag to the next energy level, indicates that the protons are taking time to accelerate from their

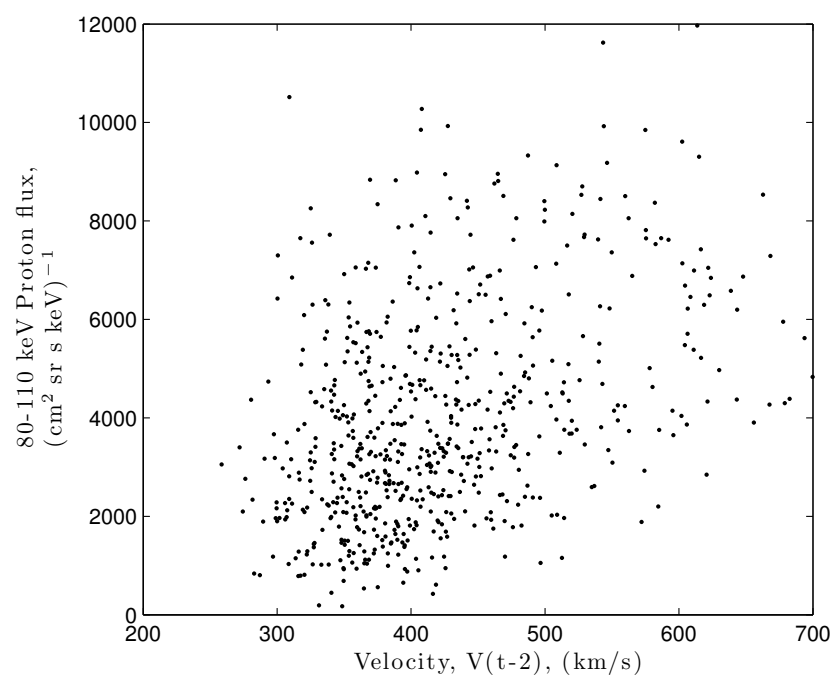

Fig. 1. A scatter plot for the daily averaged $80-110 \mathrm{keV}$ proton flux and solar wind velocity recorded two days prior.

seed energy to the higher energies observed by GOES. This would fit theories of both local and radial diffusion (Millan and Baker, 2012), where particles are accelerated over several days. However, the $350-800 \mathrm{keV}$ flux lowered to a lag of two days.

The Dst index, solar wind pressure and density all have a minor influence in controlling the fluxes between 80 and $350 \mathrm{keV}$. The current days Dst index is easily explained, since it is an indication of the strength of the ring current and the main carriers of the ring current are ions with energies from $1 \mathrm{keV}$ to a few hundred $\mathrm{keV}$ (Daglis et al., 1999). As such a higher current should mean more charged particles of these energies are trapped by the Earth's magnetic field.

The SEP have a significant ERR for the $350-800 \mathrm{keV}$ protons, which may indicate that the SEPs can penetrate into the magnetosphere to GEO for energies lower than $1 \mathrm{MeV}$. Figure 2 displays a number of SEP events observed by ACE in the top panel for the energy range $546-761 \mathrm{keV}$ and the daily averaged $350-800 \mathrm{keV}$ protons observed by GOES in the bottom panel, for the period between 18 October 2011 and 26 March 2012. For each of the peaks in SEPs, there is a corresponding peak in the proton flux at GEO on the same day.

The higher energy fluxes $(0.74-14.5 \mathrm{MeV})$ studied here are all controlled mainly by the SEP, which was established in the 1960s (Paulikas and Blake, 1969). For the 0.744.2 MeV protons, the SEP flux is coupled with the velocity, which suggests that higher solar wind speed could increase the access of the SEPs. The east and west detectors, for this channel, resulted in the same inputs. This could be due to the gyrocenters of these protons having origins that are not so far apart, and as such, the controlling parameters should not be different. Also, some of the detail may be lost with the daily averaging, such as the $1-3 \mathrm{~h}$ anisotropies reported by Rodriguez et al. (2010). 


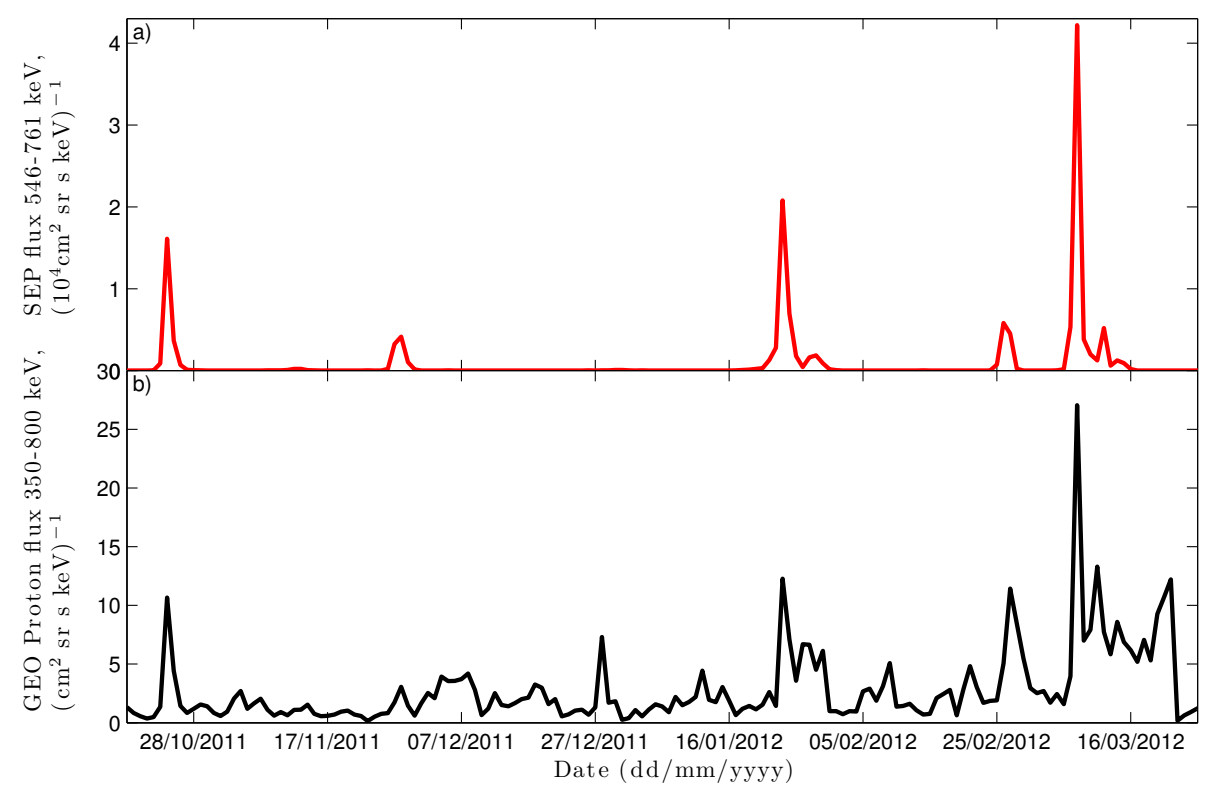

Fig. 2. Panel (a) displays the daily averaged SEP flux observed by the ACE EPAM for the energy range 546-761 keV and panel (b) shows the daily averaged 350-800 keV protons observed by GOES MAGPD, for the period between 18 October 2011 and 26 March 2012.

However, the $4.2-8.7 \mathrm{MeV}$ and $8.7-14.5 \mathrm{MeV}$ channels have differences between the east and west observations. Both channels have a higher influence from the Dst index for the fluxes inside GEO and also for the IMF-clock angle function. Similar to the inside GEO fluxes, the fluxes outside of GEO are also effected by both Dst and a southward IMF, however, the influence is smaller. Therefore, during geomagnetic storms when the IMF is predominately southward, the probability of SEPs penetrating within the GEO is increased, while the SEPs are more likely to penetrate into the region outside of GEO regardless of IMF direction.

The $>350 \mathrm{keV}$ proton fluxes are all very dependent on the current days SEP flux. As such, it will not be possible to predict the next days value because the next days SEP is required for the forecast. Even if the SEPs could be estimated from images from the Sun, it would not be enough since $350 \mathrm{keV}$ protons will take under $8 \mathrm{~h}$ to arrive at Earth. Therefore, modelling the $>350 \mathrm{keV}$ GEO proton fluxes for the purpose of an accurate forecast, will be very difficult. For the $<350 \mathrm{keV}$ proton flux the inputs are less reliant on the current days values so a model could be derived for the forecast of the next day fluxes.

\section{Conclusions}

The ERR study has shown that for energies $<800 \mathrm{keV}$, the main control parameter is the solar wind velocity, showing a similar triangular dependance observed with electron fluxes (Reeves et al., 2011). The lag of the velocity is between 23 days which indicates that the solar wind velocity supplies the seed population of protons, which is then accelerated over two days to the higher energies.

Another interesting result from the ERR analysis is that, while the solar wind velocity accounts for most of the variance for the 350-800 keV GEO proton fluxes, the SEPs have a significant contribution, $10 \%$ of the ERR. Therefore, the $350-800 \mathrm{keV}$ SEPs may have access to GEO altitudes of the magnetosphere.

For the two highest energy channels $(4.2-14.5 \mathrm{MeV})$, the IMF and Dst index enhance the access of SEPs to altitudes inside and outside of GEO. The fluxes inside GEO had more variance explained by the IMF and Dst compared to the fluxes outside of GEO. Therefore, the probability of SEPs having access to lower altitudes may increase in the case of geomagnetic storms or a southward IMF.

Producing forecast models for the GEO proton flux energies effect by SEP is currently not possible, since to forecast next day's flux at GEO would also require a forecast of the next day's SEP flux. However, for the lower energies below $350 \mathrm{keV}$, the GEO proton fluxes do not rely as much on the current day's value and therefore it is possible to to derive a forecast model that will estimate the next day's proton flux at GEO.

Acknowledgements. The authors would like to acknowledge the financial support from EPSRC, Royal Society collaboration, and ERC. RJB and OAA are grateful for support from ISSI (Bern). The Authors would also like to thank the data providers. OMNIWeb service for providing the solar wind data, ACE for providing the level 2 EPAM data, and NOAA for the GOES proton flux data at GEO.

Guest Editor M. Gedalin thanks D. Vassiliadis and one anonymous referee for their help in evaluating this paper. 


\section{References}

Balikhin, M. A., Boaghe, O. M., Billings, S. A., and Alleyne, H. S. C. K.: Terrestrial magnetosphere as a nonlinear resonator, Geophys. Res. Lett., 28, 1123-1126, 2001.

Balikhin, M. A., Boynton, R. J., Billings, S. A., Gedalin, M., Ganushkina, N., Coca, D., and Wei, H.: Data based quest for solar wind-magnetosphere coupling function, Geophys. Res. Lett., 37, L24107, doi:10.1029/2010GL045733, 2010.

Balikhin, M. A., Gedalin, M., Reeves, G. D., Boynton, R. J., and Billings, S. A.: Time scaling of the electron flux increase at geo: The local energy diffusion model vs observations, J. Geophys. Res., 117, A10208, doi:10.1029/2012JA018114, 2012.

Billings, S. A. and Voon, W. S. F.: Correlation based model validity tests for non-linear models, Int. Control J., 44, 235-244, 1986.

Billings, S. A., Chen, S., and Korenberg, M. J.: Identification of mimo non-linear systems using a forward-regression orthogonal estimator, Int. J. Control, 49, 2157-2189, 1989.

Blake, J. B., Martina, E. F., and Paulikas, G. A.: On the access of solar protons to the synchronous altitude region, J. Geophys. Res., 79, 1345-1348, doi:10.1029/JA079i010p01345, 1974.

Blake, J. B., Kolasinski, W. A., Fillius, R. W., and Mullen, E. G.: Injection of electrons and protons with energies of tens of mev into $l<3$ on 24 march 1991, Geophys. Res. Lett., 19, 821-824, doi:10.1029/92GL00624, 1992.

Boaghe, O. M., Balikhin, M. A., Billings, S. A., and Alleyne, H.: Identification of nonlinear processes in the magnetospheric dynamics and forecasting of dst index, J. Geophys. Res., 106, 30047-30066, 2001.

Boynton, R. J., Balikhin, M. A., Billings, S. A., Wei, H. L., and Ganushkina, N.: Using the narmax ols-err algorithm to obtain the most influential coupling functions that affect the evolution of the magnetosphere, J. Geophys. Res., 116, A05218, doi:10.1029/2010JA015505, 2011.

Boynton, R. J., Balikhin, M. A., Billings, S. A., Reeves, G. D., Ganushkina, N., Gedalin, M., Amariutei, O. A., Borovsky, J. E., and Walker, S. N.: The analysis of electron fluxes at geosynchronous orbit employing a narmax approach, J. Geophys. Res. Space Physics, 118, 1500-1513, doi:10.1002/jgra.50192, 2013.

Burton, R. K., McPherron, R. L., and Russull, C. T.: An empirical relationship between interplanetary conditions and dst, J. Geophys Res., 80, 4204-4214, 1975.

Daglis, I. A., Thorne, R. M., Baumjohann, W., and Orsini, S.: The terrestrial ring current: Origin, formation, and decay, Rev. Geophys., 37, 407-438, doi:10.1029/1999RG900009, 1999.

Hanser, F. A.: Eps/hepad calibration and data handbook, Technical report, Tech. Rep. GOESN-ENG-048D, Assurance Technol. Corp., Carlisle, Mass., 2011.

Horne, R. B., Thorne, R. M., Glauert, S. A., Albert, J. M., Meredith, N. P., and Anderson, R. R.: Timescale for radiation belt electron acceleration by whistler mode chorus waves, J. Geophys. Res., 110, A03225, doi:10.1029/2004JA010811, 2005.
Hudson, M. K., Kotelnikov, A. D., Li, X., Roth, I., Temerin, M., Wygant, J., Blake, J. B., and Gussenhoven, M. S.: Simulation of proton radiation belt formation during the march 24, 1991 ssc, Geophys. Res. Lett., 22, 291-294, doi:10.1029/95GL00009, 1995.

Ivanova, T. A., Kuznetsov, S. N., Sosnovets, E. N., and Tverskaya, L. V.: Dynamics of the low-latitude limit of penetration of lowenergy solar protons into the magnetosphere, Geomagn. Aeron., Engl. Transl., 25, 5-8, 1985.

Kan, J. R. and Lee, L. C.: Energy coupling function and solar windmagnetosphere dynamo, Geophys. Res. Lett., 6, 577-580, 1979.

Leontaritis, I. J. and Billings, S. A.: Input-output parametric models for non-linear systems part i: Deterministic non-linear systems, Int. J. Control, 41, 303-328, 1985a.

Leontaritis, I. J. and Billings, S. A.: Input-output parametric models for non-linear systems part ii: Stochastic nonlinear systems, Int. J. Control, 41, 329-344, 1985b.

Li, X., Roth, I., Temerin, M., Wygant, J. R., Hudson, M. K., and Blake, J. B.: Simulation of the prompt energization and transport of radiation belt particles during the march 24, $1991 \mathrm{ssc}$, Geophys. Res. Lett., 20, 2423-2426, doi:10.1029/93GL02701, 1993.

Millan, R. M. and Baker, D. N.: Acceleration of particles to high energies in earth's radiation belts, Space Sci. Rev., 173, 103-131, doi:10.1007/s11214-012-9941-x, 2012.

Paulikas, G. A. and Blake, J. B.: Penetration of solar protons to synchronous altitude, J. Geophys. Res., 74, 2161-2168, doi:10.1029/JA074i009p02161, 1969.

Reeves, G. D., Morley, S. K., Friedel, R. H. W., Henderson, M. G., Cayton, T. E., Cunningham, G., Blake, J. B., Christensen, R. A., and Thomsen, D.: On the relationship between relativistic electron flux and solar wind velocity: Paulikas and blake revisited, J. Geophys. Res., 116, A02213, doi:10.1029/2010JA015735, 2011.

Richard, R. L., El-Alaoui, M., Ashour-Abdalla, M., and Walker, R. J.: Interplanetary magnetic field control of the entry of solar energetic particles into the magnetosphere, J. Geophys. Res., 107, SSH 7-1-SSH 7-20, doi:10.1029/2001JA000099, 2002.

Rodriguez, J. V., Onsager, T. G., and Mazur, J. E.: The east-west effect in solar proton flux measurements in geostationary orbit: A new goes capability, Geophys. Res. Lett., 37, L07109, doi:10.1029/2010GL042531, 2010.

Roeder, J. L. and Fennell, J. F.: Differential Charging of Satellite Surface Materials, IEEE T. Plasma Sci., 37, 281-289, doi:10.1109/TPS.2008.2004765, 2009.

Schulz, M. and Lanzerotti, L. J.: Particle diffusion in the radiation belts, Physics and Chemistry in Space, Berlin: Springer, 1974.

Vampola, A. L. and Korth, A.: Electron drift echoes in the inner magnetosphere, Geophys. Res. Lett., 19, 625-628, doi:10.1029/92GL00121, 1992.

van Allen, J. A.: Cosmic-ray observations in earth satellites, in: Scientific Uses of Earth Satellites, edited by: van Allen, J. A., 1958. 